Vergleicht man nun die Reihenfolge der Elemente, wie sie durch die Kernladung (oder „Ordnungszahl“ im Sinne M o seley) bedingt ist mit derjenigen, die das nach steigendem A.G. angeordnete System darstellt, so mitssen, grob betrachtet, beide Folgen identisch sein. Der Schwerpunkt liegt aber oben auf dem w-Zeichen (ungefăhr gleich). Hierdurch entstehen geringfügige Abweichungen der Moseleyschen von der nach steigendem A.G. geordneten Reihe. Es erscheint bei Moseley $\mathrm{K}$ binter A, Ni hinier $\mathrm{Co}$, Te hinter $J$, wie das chemische Verhalten dieser Elemente es im Gegensatz zum A.G. fordert.

Ein kleiner Schritt noch und wir haben, was wir suchen: den Ersatz für das A.G. als ordnendes Prinzip im System der Elemente.

Wir vermuten inn in der Moseleyschen Ordnungszahl.

Soll diese unsere Erwartungen erfullen, so muß sie auch den verschiedenen Bleiarten ihre Plätze anweisen. Man hat also das charakteristische Röntgenspektrum dieser Körper zu untersuchen um ihre Ordnungszahl zu finden. Diese Messungen wurden von Siegbahn und Stenstróm durchgeführt und zeitigten das Resultat, dab allen drei Bleiarten dieselbe Ordnungszahl zukommt, wie es bei der Gleichheit der Eigenschaften diestr Elemente zu erwarten ist.

Wir sind nun am Ziele: wir ordnen die Elemente nach der Moseleyschen Ordnungszahl und erhalten dadurch eine auf physikalischer Grundlage aufgebaute Reihenfolge, die sich mit der empirisch vom Chemiker aufgestellten, dem periodischen System vollkommen deckt.

Das Unbefriedigende, was in der Empirie des periodischen Systems lag, ist damit bis zu einem gewissen Grade behoben. Immerhin bleibt noch aufzuklären, woher nun die periodische Abhängigkeit der chemischen Eigenschaften der Elemente gerade von ihrer Kernladung $(=$ Ordnungszahl) rührt.

In diesem Zusammenhange werden uns nun auch die obengenannten radioaktiven Verschiebungssätze verständlich.

1. Eine $\alpha$-Strahlenumwandlung besteht darin, daß der Korper aus seinem Atomkern ein $\alpha$-Teilchen, also den (doppelt positiv) geladenen Kern eines Heliumatoms verliert. Ein herangeschleudertes $\alpha$-Teilchen entzieht also dem Atomkern zwei positive Ladungseinheiten. Nach der Umwandlung hat also der Kern zwei positive Ladungseinheiten weniger, seine Ordnungszahl ist um zwei Einheiten verringert.

2. Bei der $\beta$-Strablenumwandlung wird ein Elektron (einfach negativ geladenes Teilchen) ausgesandt. Der übrigbleibende Kern erscheint also mit einer einfachen + -Ladungseinheit mehr als vor dem Zerfall, seine Ordnungszahl ist um eine Einheit gestiegen. Die Ứbereinstimmung dieser Folgerungen mit den experimentell festgestellten Verschiebungssätzen bestätigt die Richtigkeit der Atomtheorie.

Das neue System zeigt ein Neues, Iämlich, daß an eine Stelle mehrere Körper (ich vermeide den Ausdruck „Elemente") zu stehen kommen.

Solcbe Körper nennt man "Isotopen“ und die Gruppe, zu der sie zusammentreten, eine "Plejade“.

Solcher Plejaden hat man nun noch mehrere gefunden: beim TI, Bi, Po, Em, Ba, Ac, Th, Pa, U.

Welche Bedeutung das Herausschälen des Isotopenbegriffes für unser Welt hild hat, ersehen Sie daran, daß es nunmehr möglich ist, die Abweichungen von der Ganzzahligkeit der A.G.., die dem alten Herrn Prout fast die Unvergeßllichkeit gekostet hätten, durch Isotopie zu erklären.

Haben wir nämlich ein Isotopenyemisch vor uns, so können wir die einzelnen Isotopen nicht voneinander trennen, sehen also das Gemisch fïr ein "Element" th. Die Bestandteile haben aber sehr wobl verschiedenes A.G. Bestimmen wir das A.G. des Gemisches, so werden wir das Mittel der A.G. der Bestandteile finden, und dies Mittel ist sicher nicht (oder nur zufällig) ganzzahlig, selbst wenn den Bestandteilen ganzzalliges A.G. zukommt.

Soweit war Prout no $h$ nicht vergessen, als daß nicht alsbald nach der Aufstellung des Isotopenbegriffes eine Reihe von Forschern diese Folgerungen gezogen hätten.

Es gilt jetzt, von einem Element, von dem man vermutet, daß es ein Isotopengemisch darstellt - also vornehmlich die Elemente mit gebrochenem A.G., wie z. B. Chlor - nachzuweisen, daß es in der Tat aus einem Gemisch besteht. Die Bestandteile müssen dann getrennt werden und ihr A.G. einzeln bestimmt werden.

Ganz unmöglich ist das nicht, aber sehr schwierig.

Man kann zur Trennung der Isotopen im Prinzip alle die physikalischen Vorgänge verwenden, in denen das A.G. eine maßgebende Rolle spielt, also vor allem die Diffusion. Es ist ja

Diffusionsgeschw: $=c_{\sqrt{D i c h t e}}=$ (In der Dichte steckt das Atomgewicht.)

Eis haben in allerneuester Zeit Versuche von Harkins und Broeker, die schon jahrelang im Gang sind und bei denen täglich $1000 \mathrm{l} \mathrm{HCl}$ fraktioniert wurden, zur Zerlegung des $\mathrm{Cl}$ in drei Isotopen geführt, deren $A . G$. bestimmt wurde

$$
35,37 \text { und } 39 \text { (wenig). }
$$

Auch Jod soll zerlegt sein (Kohlweiler).

$\mathrm{Da} H \mathrm{H}$ und $\mathrm{O}$ keine Irotopengemische sind, haben Stern und Volmer durch Diffusionsversuche nachewiesen. Hevesy destillierte unter gewissen Bedingungen $\mathrm{Hg}$ fraktioniert und erhielt Proben, deren Dichte sich um $3 \%$ voneinander unterschieden.

Soweit die effentive 'Trennung der [sotopen.

Leichter ist es, Isotopen in einem Gemisch nur nachzuweisen. Das könnte durch spektroskopische Messungen gelingen, denn Merton, ferner Harkins und Aronberg haben nachgewiesen, daß z. B. die Blei-Isotopen verschiedene Emissionsspektren haben. Der Unterschied beträgt für die $\mathrm{Pb}$-Linie

$$
\lambda=4058 \begin{aligned}
& 0,005 \pm 0,0007 \stackrel{\circ}{\mathrm{A} E} \\
& \text { (bei Pb und Pb) } \\
& 207 \quad 206
\end{aligned}
$$

Die eleganteste Methode zur Erkennung der Isotopen und gleichzeitigen Bestimmung ihres Atomgewichts ist 'zweifellos die von Thomson erdachte, von Aston ausgestaltete Kanalstrahlenanalyse im sogenannten Messungsspektrographen. Auf die Methode will ich hier nicht eingehen; sie beruht darauf, daß die Ablenkung der Kanalstrablen im Magnetfeld umgekehrt proportional ist $\mathrm{m} \mathrm{v}$, die im elektrischen Feld umgekehrt $\mathrm{m} \mathrm{v}^{2}(\mathrm{~m}=$ Masse, $\mathrm{v}=$ Geschwindigkeit der Teilchrn)

Die Tafel zeigt alle Ergebnisse uber Isotopenforschung. Die meisten

\begin{tabular}{|c|c|c|c|}
\hline $\begin{array}{l}\text { Ord- } \\
\text { nungs- } \\
\text { zahl }\end{array}$ & Symb. & Atgew. & $\begin{array}{l}\text { Atgew. der } \\
\text { Isotopen }\end{array}$ \\
\hline $\begin{array}{l}1 \\
2 \\
3 \\
\end{array}$ & $\begin{array}{l}\mathrm{H} \\
\mathrm{He} \\
\mathrm{Li}\end{array}$ & $\begin{array}{l}1,008 \\
4,00 \\
6,94\end{array}$ & 6,7 \\
\hline $\begin{array}{r}5 \\
6 \\
7 \\
8 \\
9 \\
10 \\
11\end{array}$ & $\begin{array}{l}\mathrm{B} \\
\mathrm{C} \\
\mathrm{N} \\
\mathrm{O} \\
\mathrm{F} \\
\mathrm{Ne} \\
\mathrm{Na}\end{array}$ & $\begin{array}{l}11,0 \\
12,0 \\
14,0 \\
16,0 \\
19,0 \\
20,2 \\
23,0\end{array}$ & $20(90 \%) 22(10 \%)$ \\
\hline $\begin{array}{l}14 \\
15 \\
16 \\
17 \\
18 \\
19\end{array}$ & $\begin{array}{l}\mathrm{Si} \\
\mathbf{P} \\
\mathrm{S} \\
\mathrm{Cl} \\
\mathrm{A} \\
\mathrm{K}\end{array}$ & $\begin{array}{l}28,3 \\
31,0 \\
32,06 \\
35,46 \\
39,88 \\
39,10 \\
\end{array}$ & $\begin{array}{l}28,29,30(?) \\
\text { überw. } 32 \\
35(3-4 \text { fach) } 37,39(?) \\
40,36 \\
39,41\end{array}$ \\
\hline 33 & As & 75 & 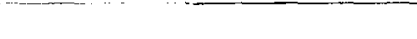 \\
\hline $\begin{array}{l}35 \\
36 \\
37\end{array}$ & $\begin{array}{l}\mathrm{Br} \\
\mathrm{Kr} \\
\mathrm{Rb}\end{array}$ & $\begin{array}{l}79,92 \\
81,92 \\
85,45\end{array}$ & $\begin{array}{l}79,81 \\
78,80,82,83,84,86 \\
85,87\end{array}$ \\
\hline $\begin{array}{l}53 \\
54 \\
55\end{array}$ & $\begin{array}{l}J \\
\mathrm{X} \\
\mathrm{Cs}\end{array}$ & $\begin{array}{l}126,92 \\
130,2 \\
132,8\end{array}$ & $129,131,132,134,136$ \\
\hline $\begin{array}{l}80 \\
81\end{array}$ & $\begin{array}{l}\mathrm{Hg} \\
\mathrm{TI}\end{array}$ & $\begin{array}{l}200,6 \\
204,0\end{array}$ & $\begin{array}{l}197 \text { bis } 200,202,204 \\
204,206,208,210\end{array}$ \\
\hline $\begin{array}{l}82 \\
83 \\
84 \\
85 \\
86 \\
87 \\
88 \\
89 \\
90 \\
91 \\
92\end{array}$ & $\begin{array}{l}\mathrm{Pb} \\
\mathrm{Bi} \\
\mathrm{Po} \\
? \\
\mathrm{Sm} \\
? \\
\mathrm{Ra} \\
\mathrm{Ac} \\
\mathrm{Th} \\
\mathrm{Pa} \\
\mathrm{U}\end{array}$ & $?$ & $\begin{array}{l}206,207,2,208,210,212,214 \\
208,210,212,214 \\
210,212,214,216,218 \\
218,220,222 \\
\\
224,225,97 ; 228 \\
226,228 \\
226,228,230 ; 232,15 ; 234 \\
230,234 \\
234,238,2\end{array}$ \\
\hline
\end{tabular}
(bis einschl. Hg) stammen von Aston, mit Bestätigung bei Chlor durch die direkten Messungen von Harkins. Die Isotopengemische hoberen Atomgewichts als $\mathrm{Hg}$ sind von der Radioaktivität her bekannt.

Dem Analytiker möchte ich zum Schluß etwa aufsteigende Zweifel an der ferneren Brauchbarkeit seiner Küsterschen Tabellen zerstreuen. Solange Sie mit den gewöhnlichen Reagentien hantieren, an denen ja die Tabellen berechnet worden sind, ist keine Besorgnis von nöten. Daß Sie mit mittleren Molekulargewichten von Gemischen rechnen können, zeigt Ihnen jede Dampfdichtebestimmung, bei der Sie ja das Molekulargewicht der Luft - also eines unzweifelhaft aus den verschiedensten Bestandteilen zusammengesetzten Gemischs - im Mittel zu 28,8 ansetzen und zu vernünftigen Resultaten kommen.

Vorsicht ist erst am Platze, wenn sie mit Uranblei arbeiten. Sie dürfen dann nicht die Berechnungen so führen, als hätten Sie $\mathrm{Pb}$ vor sich. Vorsicht wäre auch nötig, wenn etwa die Amerikaner Salzsäure in den Handel brächten, die aus Harkinsschem getrennten Chlor hergestellt wäre. Dies ist aber kaum zu befürchten, denn der Preis dieser Salzsäure wäre doch wohl zu hoch.
[A. 222.]

\section{Methode zur direkten Wasserbestimmung in Mischsäuren.}

Von E. BerL und W. v. BOLtenstern.

(Eingeg. 29.9. 1921.)

Bei der Cellulosenitraterzeugung bildet nach Untersuchungen, hauptsächlich von Lunge und seiner Schule (L unge und Weintraub, Zeitschr. f. angew. Chem. 12, 441; 1899; Lunge und B ebie, ebenda 14, 511; 1901; Berl und Klaye, Zeitschr. f. d. ges. Schieß- und Sprengstoff wesen 2,382;1907) und anderen Forschern, unter andern Rassow 
und v. Bongé (diese Zeitschr. 21, 732 [1908]) der absolute Wassergehalt der Mischsäuren den wichtigsten Faktor, der den Stickstoffgehalt der erzeugten Nitrocellulosen bestimmt. Ähnlich, wenn auch nicht so ausschlaggebend, liegen die Verhältnisse bei der Erzeugung von Glycerintrinitrat und von Polynitroderivaten aromatischer Kohlenwasserstoffe und deren Abkömmlingen. Hiernach ergibt sich die Forderung, diesen für die Erreichung einer gewollten Qualität wichtigsten Bestandteil der Mischsäuren möglichst direkt zu bestimnen. Durch keine der bisher bekannten Analysenmethoden (vgl. Lunge-Berl, Chem.-techn. Untersuchungsmethoden 1921, Bd. I, S. 878 und Berl und v. Boltenstern, Zeitschr. f. angew. Chem. 34, 19; 1921) ist diese technisch wichtige Forderung erfibllt, da bei diesen sich in direkt der Wassergehalt durch Abziehen der direkt bestimmten Einzelbestandteile von 100 ergibt.

Im folgenden wird eine Methode beschrieben, die auf der Messung der Verdünnungswärme von Mischsäuren mit Wasser beruht. Diese ist innerhalb bestimmter Grenzen abbängig vom Wassergehalt der Säuren, wodurch dieser direkt bestimmbar wird. In vorliegender Arbeit wird die experimentelle Durchführung dieser Analysenmethode geschildert. Über die rein rechnerische Behandlung des Problems soll im Zusammenhang mit anderen Messungen später berichtet werden.

Das Prinzip der hier eingeschlagenen calorimetrischen Methode hat Howard (Journ. Soc. Chem. Ind. 29, 3; 1910) bei der Analyse von Oleum angegeben. Weitere Untersuchungen über Oleumanalyse auf gleicher Grundlage rühren von $\mathrm{Ri} \mathrm{ch}$ m ond und M e rre y weth er (Chem. Zentralbl. 1917, II, 771) und Curt is und Miles (ebenda 1920, IV, 63) her.

Die Apparatur ${ }^{\prime}$ ) (Fig.1) besteht aus einem versilberten, gut evakuierten zylindrischen Weinholdgefäß (D) von mindest $40 \mathrm{~mm}$ Durchmesser

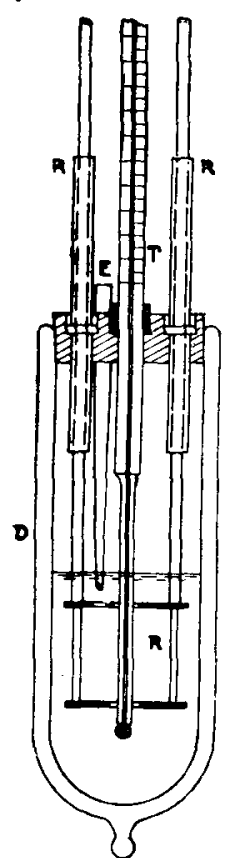

Fig. 1, das Gefäß in einen Kasten mit Kieselgur, Baumwolle oder ähnlichem Isoliernıaterial eingepackt. Als Verschluß des Gefäßes dient ein paraffinierter Korkstopfen oder besser noch ein eingeschliffener Glasstopfen. Dieser hat vier Bohrungen, eine für das Thermometer (T), eine zweite zum Einlaufenlassen der Mischsäure (E) und zwei für den Rührer (R). Das Thermometer soll in ${ }^{1} 10^{\circ} \mathrm{C}$ genau eingeteilt sein, einen Meßbereich ron etwa $0^{0}-50^{\circ} \mathrm{C}$ besitzen und mindestens bis zur halben Tiefe der Flüssigkeit in das Gefäß bineinreichen. Die Mischsäure läßt man aus einer Glashahnbürette mit möglichst feiner Auslaufspitze durch das Einlaufrohr (E) in das Gefäß einlaufen. Das Einlaufrohr, das oben etwas aus dem Kork- oder Glasstopfen herausragt, ist nach unten kapillar ausgezogen und taucht einige Millimeter in die Flüssigkeit ein. Der Rührer (R) besteht aus zwei Glas- oder Aluminiumplättchen, die für das Einlaufrolır und das Thermometer Anssparungen haben und miteinander starr verbunden sind. Um jede Reibung innerhalb der A ppiaratur zu vermeiden. erbält der Rührer doppelte Führung in zwei Glasiöhren. Der Antrieb des Rïhrwerks geschieht durch einen Exzenter, wie man ihn bei den Calorimetern anwendet. Die Tourenzahl soll 60-80 in der Minute betragen. Es ist darauf zu achten, daß der Rührer die Flüssigkeit rasch und gut durchmischt und keinerlei Reibungen innerhalb der Apparatur auftreten.

Die Arbe itsweis e ist tolgende: Man füllt mit

einer Pipette 200 cem destilliertes Wasser vor einer Temperatur, die etwa um die Hailfte der zu erwartenden Temperaturerhöhung unterhalb der Zimmertemperatur liegt, in das trockene $W($ in h old gefäß ein, setzt die Apparatur zusammen und stellt das Rührwerk an. Nach $3-4$ Minuten kann der Versuch beginnen. Der Temperatırgang wird mit einer Lupe am Thermometer von Minute zu Minute genau abgelesen und notiert. Ist er regelmäbig geworden, so kann der eigentliche Versuch beginnen. Man laikt nun aus der l3ürette die Menge von $20,0 \mathrm{ccm}$ der zu analysierenden Mischsiaure, die so weit wie möglich dieselbe Temperatur wie das Wasser im Weinholdgefäß h haben muß, langsam zufließen und zwar so. daß die Gesamteinlaufzeit 75 Sekunden beträgt. Nunmehr wird das Thermometer wieder von Minute zu Minute genau abgelesen, bis der Temperaturgang wieder einige Minuten gleichmäßig geworden ist. Die Berechnurg des wahren Temperaturunterschiedes geschieht in der gleichen Weise wie bei der Heizwertbestimmung mit der Mahler-Berthelot-Bombe (vgl. Lunge-Berl, Chem.-techn. Untersuchungsmethoden 1921. Bd. I, S. 438).

Beispiel für eine Messung:

$200 \mathrm{~cm} \mathrm{Wasser}$.

$20,0 \mathrm{ccm}$ Mischsäure : $\frac{\mathrm{H}_{2} \mathrm{SO}_{4}}{\mathrm{HNO}}=: 3: 1 ; 0,1^{\circ !}, \mathrm{N}_{2} \mathrm{O}_{3} ;{ }^{\prime}$ Temp. $14,98^{\circ} \mathrm{C}$

Einlaufzeit der Säure: $75^{\prime \prime}$

\begin{tabular}{|c|c|c|c|}
\hline Vorversuch: & $\begin{array}{ll}0^{\prime} & 15,26 \\
1^{\prime} & 15,28 \\
2^{\prime} & 15,30 \\
3^{\prime} & 15,32\end{array}$ & $\begin{array}{r}+0,02 \\
+0,02 \\
+0,02\end{array}$ & $\begin{array}{l}\text { im Mittel: } \\
\quad+0,0^{0}{ }^{0}\end{array}$ \\
\hline
\end{tabular}

1) Zu beziehen von Elirbardt \& Metzger. Darmstadt.
Hauptversuch:

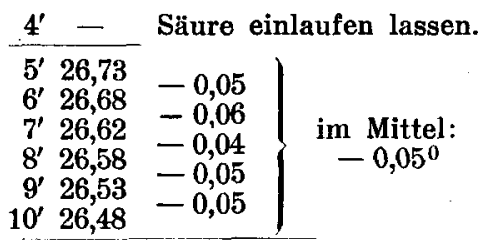

$$
\begin{aligned}
& \text { Scheinbare Temperaturdifferenz: } \quad 26,73^{\circ} \mathrm{C} \\
& \frac{-15.32^{\circ} \mathrm{C}}{11,41^{\circ} \mathrm{C}} \\
& \text { Korrektur für Minute } 3-4=\frac{-0,02+0,05}{2}=+0,01^{\circ} \mathrm{C} \\
& \begin{array}{l}
4-5=\quad+0,05=+0,05^{\circ} \mathrm{C} \\
+11,47^{\circ} \mathrm{C}
\end{array}
\end{aligned}
$$

Diese Temperaturerhơhung entspricht nach Eichkurve.....einem Wassergehalt von ..... o/

Die Eichkurve stellt man sich zweckmäßig in der Weise her, daß man von einer möglichst nitrosefreien und von organischer Substanz freien Säure, die vorher mehrmals genau analysiert ist (vgl. Berl und v. Boltenstern, Zeitschr. f. angew. Chem. 34, 19; 1921), ausgeht. Durch Verdünnen einer genau gewogenen Menge dieser Säure mit bekannten Wassermengen stellt man sich Säuren von verschiedenem Wassergehalt her. Durch Kontrollanalyse überzeugt man sich bei einigen Proben von der Richtigkeit der berechneten Zusammensetzung. Diese Methode der Säureherstellung hat den Vorteil, daß dabei das relative Verhältnis von $\mathrm{H}_{2} \mathrm{SO}_{4}$ zu $\mathrm{HNO}_{3}$ nicht geändert wird. Nunmehr werden mit diesen einzelnen Säuren Messungen in genau derselben Weise, wie oben beschrieben, durchgeführt. Die ermittelten Temperaturpunkte werden in ein Koordinatensystem, auf dessen Ordinate die Celsiusgrade, auf dessen Abszisse der Wassergehalt in Prozenten aufgetragen ist, eingetragen und durch eine Kurve miteinander verbunden. Aus dieser Eichkurve lassen sich dann die Versuchswerte genau ablesen.

In der Praxis wird man normale Betriebssäuren mit genau bekannten Gehalten an $\mathrm{N}_{2} \mathrm{O}_{3}$ und organischer Substanz den Eichkurven zugrunde legen.

Der Nitrosegebalt der Mischsäuren ist in der Weise zu berücksichtigen, daß für jedes Prozent $\mathrm{N}_{3} \mathrm{O}_{3}$, das mehr oder weniger in der zu analysierenden Säure vorbanden ist als in der zur Herstellung der Eichkurve verwendeten Säure, $1 \%$ von dem gefundenen Wassergehalt in Abzug zu bringen oder zuzuzählen ist.

Für verschiedene Säureverhältnisse $\mathrm{H}_{2} \mathrm{SO}_{4}: \mathrm{HNO}_{3}$ sind verschiedene Eichkurven aufzustellen. $Z$. B. stellt man sich in der Praxis der Cellulosenitratfabrikation zweckmäßig eine Eichkurve für eine Durchschnittsnitriersäure und eine Durchschnittsabgangssäure her.

Der Gehalt an organischer Substanz beeinflußt die Messungsergebnisse nicht, $d$. h. Säuren mit gleichem Säureverhältnis und gleichem Wassergehalt geben bei Anwesenheit und Abwesenheit von organischer Substanz gleiche Wärmetönungen. Darin liegt gegenüber den früher angewandten Methoden der Mischsäureuntersuchung, be denen die organische Substanz als Wasser oder $\mathrm{N}_{2} \mathrm{O}_{3}$ angesehen wurde (vgl. Berl und v. Boltenstern, Zeitschr. f. angew. Chem. 34, 19 1921), ein großer Vorteil.

Die Genauigkeit der Messungen ist groß. Bei Verwendung eines in $1 / 10^{\circ} \mathrm{C}$ eingeteilten geeichten Thermometers lassen sich mit der Lupe $0,03^{\circ} \mathrm{C}$ genau ablesen, diese entsprechen für eine norma zusammengesetzte, wasserhaltige Nitriersäure einem durchschnittlichen Wassergehalt von $0,05-0,06^{\circ} \%$ Man erzielt also eine Genauigkeit, wie sie bei den indirekten Methoden niemals erreicht wird. Durch Änderung der Versuchsbedingungen läßt sich die Genauigkeit noch steigern, je nach der Menge der Mischsäure, die man in das Wasser einlaufen läßt. Es empfiehlt sich aber, nicht zu große Mengen von Mischsäuren zu verwenden, weil bei großen Temperaturerhöhungen die Abstrablung der Apparatur zu groß wird, worunter die Genauigkeit der Messung leidet.

Ein Hauptvorteil dieser Methode liegt neben der Möglichkeit der direkten Wasserbestimmung besonders noch darin, daß die Wasserbestimmung sich in sehr kurzer Zeit durchführen läßt, was besonders dann von Vorteil ist, wenn es sich um die rasche Ausführung einel großen Zahl von Säureanalysen handelt, wie sie z. B. bei der Erzeugung von Cellulosenitrat täglich vorgenommen werden müssen.

\section{Versuchsreihe:}

Versuche.

Aufstellung einer Eichkurve für Mischsäure, frei von organischer Substanz. Säureverhältnis $\mathrm{H}_{2} \mathrm{SO}_{4}: \mathrm{HNO}_{3}$ ungefähr $3: 1$. $2500 \mathrm{~g}$ Mischsäure wurden aus 3 Teilen konzentrierter Schwefelsäure und 1 Teil konzentrierter $\mathrm{HNO}_{3}$ hergestellt.

Die wiederholte genaue Analyse ergab:

$72,78 \%{ }_{0} \mathrm{H}_{2} \mathrm{SO}_{4} ; 23,26 \% \mathrm{HNO}_{3} ; 0,12 \% \mathrm{~N}_{2} \mathrm{O}_{3} ; 3,84 \% \%_{0} \mathrm{H}_{2} \mathrm{O}$ und

$\mathrm{H}_{2} \mathrm{SO}_{4}: \mathrm{HNO}_{3}=3,13: 1$.

Nunmebr wurden 20 Proben dieser Säure von je $125,0 \mathrm{~g}$ ausgewogen. $\mathrm{Zu}$ den einzelnen Proben wurden steigend $1 \times 1,5$ bis $20 \times 1,5 \mathrm{ccm}$ Wasser aus einer Bürette zugegeben und die prozentische Zusammensetzung der Säure errechnet. 
Die Messungen mit diesen Säureproben wurden in genau derselben Weise, wie oben beschrieben, durchgeführt.

Die gefundenen Werte sind in Fig. 2 zusammengestellt.

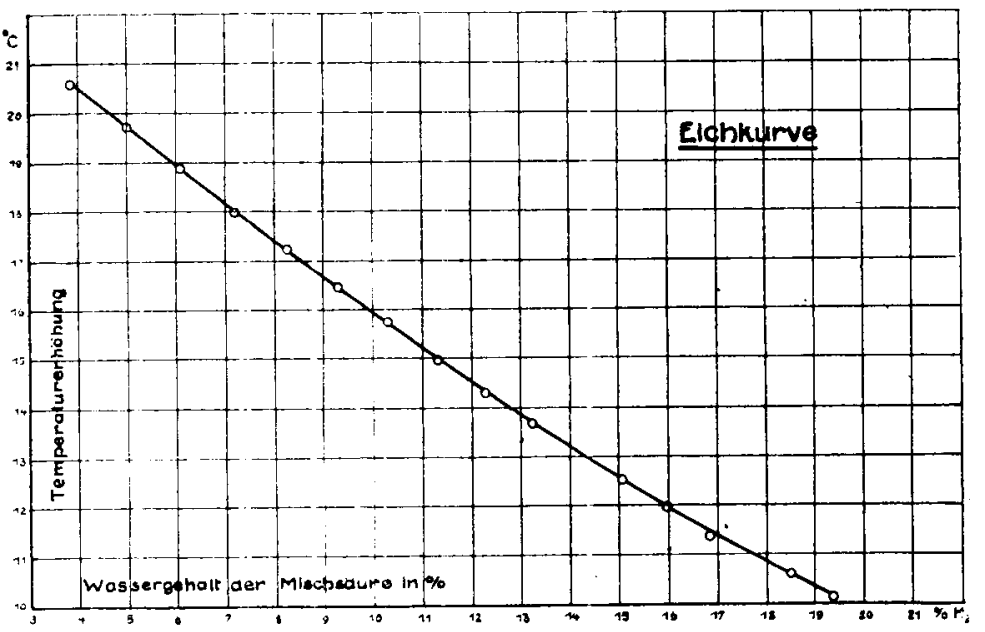

Fig. 2.

2. Versuchsreihe:

Änderung des Säureverhältnisses $\mathrm{H}_{2} \mathrm{SO}_{4}$ : $\mathrm{HNO}$

$$
\mathrm{H}_{2} \mathrm{SO}_{4}: \mathrm{HNO}_{3} \quad \% \mathrm{~N}_{2} \mathrm{O}_{3}
$$

l. $3,26: 1$

0,25

II. $3,16: 1$

III. $2,09:$

IV. $4,17: 1$

0,09

0,20

0,10 .

Die Ergebnisse sind in Fig. 3 graphisch wiedergegeben. Es geht

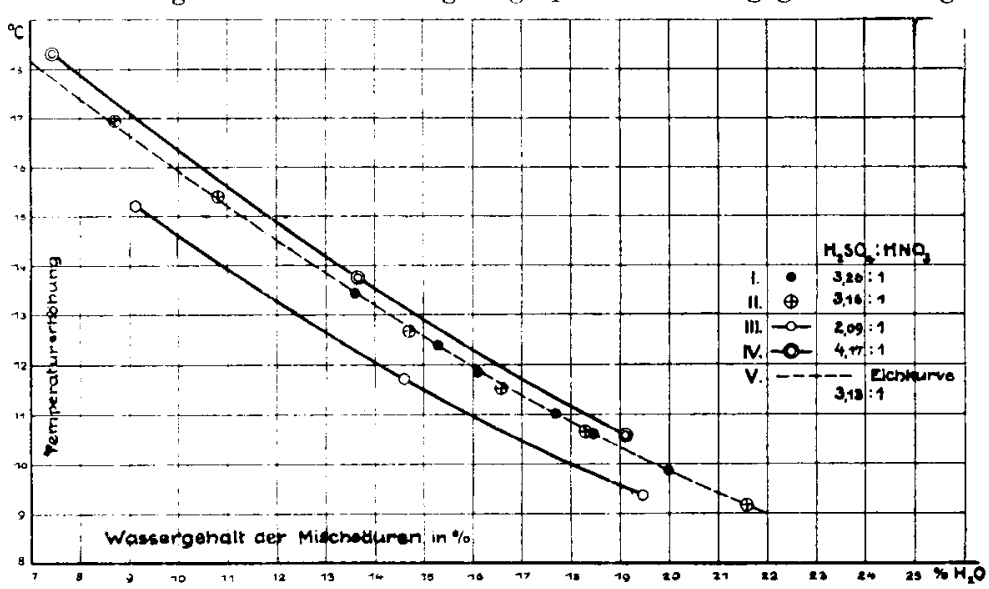

Fig. 3.

daraus hervor: Je größ3er das Verhältnis $\mathrm{H}_{2} \mathrm{SO}_{4}: \mathrm{HNO}_{3}$, um so größer werden bei gleichbleibendem Wassergehalt der Mischsäuren die Verdünnungswärmen, denınach die festgestellten Temperaturerhöhungen. Mit steigendem Wassergehalt der Säuren nehmen diese Temperaturerhöhurgen $a b$.

Die Kurve $V$ in Fig. 3 gibt nochmals die Werte der 1. Versuchsreihe zum Vergleich. Miın kann daraus ersehen, daß geringe Änderungen im Säureverhältnis keine nennenswerten Abweichungen hervorrufen.

3. Versuchsreihe:

Versuche mit stark nitrosen Säuren.

I. $\mathrm{H}_{2} \mathrm{SO}_{4}: \mathrm{HNO}_{3}$ (als $\mathrm{N}_{2} \mathrm{O}_{3}$ freie $\mathrm{HNO}$

berechnet) $=2,93: 1 ; \quad 1,95 \% \mathrm{~N}_{2} \mathrm{O}_{3}$.

II. $\mathrm{H}_{2} \mathrm{SO}_{1}: \mathrm{HNO}_{3}$ (als $\mathrm{N}_{2} \mathrm{O}_{3}$ freie $\mathrm{HNO}_{3,}$ berechnet) $=3,05: 1: 2,76-2,566^{\circ}:{ }_{0} \mathrm{~N}_{2} \mathrm{O}_{3}$.

III. $\mathrm{H}_{2} \mathrm{SO}_{1}: \mathrm{HNO}_{3}$ (als $\mathrm{N}_{2} \mathrm{O}_{i j}$ freie $\mathrm{HNO}_{3}$

berechnet) $=3,09: 1 ; 1,44-1,29^{\circ} \mathrm{O}_{0} \mathrm{~N}_{2} \mathrm{O}_{3}$.

Vergleicht man die für diese Säuren gefundenen Werte (Fig. 4) mit den Werten der 1. Versuchsreihe (in der Figur gestrichelt eingezeichnet), so fällt auff, daß sie um so tiefer unterhalb der Versuchsergebnisse mit nitrosearmen Säuren liegen, je höher ihr Gehalt an N.O, ist. Dies bedeutet, daf ein Gehalt anl, vorzugsweise als Nitrosylschwefelsäure $\left(\mathrm{S}_{5} \mathrm{NH}\right)$, gebundenem $\mathrm{N}_{2} \mathrm{O}_{; ;}$den Wassergehalt zu hoch erscheinen läßt.

Wie aus Fig. 5 hervorgeht, besteht annähernd die Beziehung, daß je $11 \% \mathrm{~N}, \mathrm{O}$, den Wassergehait um 1": zu hoch erscheinen läßt.

Bei Zugrundelegung einer Eichkurve mit Säuren von gleichem Nitrosegehalt wird eine Korrektur für den $\mathrm{N}_{2} \mathrm{O}_{3}-$ oder $\mathrm{SO}_{5} \mathrm{NH}-$ ) Gehalt nicht vorgenommen.

4. Versuchsreihe:

Untersuchungen über den Einflug von organischer Substanz.
Verschiedene Mischsäuren wurden mit feingepulverter, kristallisierter Oxalsäure versetzt. Das Säureverhältnis wurde konstant gehalten.

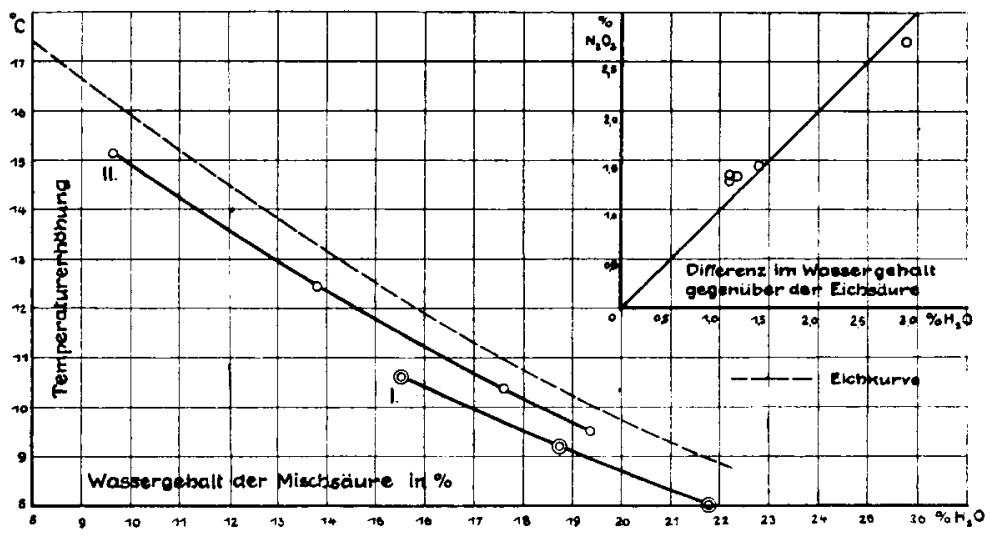

Fig. 4 u. 5 .

Die Zusammensetzung der verschiedenen Säuren war:

\begin{tabular}{c|c|c}
\hline & $\mathrm{H}_{2} \mathrm{SO}_{4}: \mathrm{HNO}_{i s}$ & $(\mathrm{COOH})_{2}$ \\
\hline 1. & $3,16: 1$ & 0,01 \\
2. & $"$ & 1,00 \\
3. & $"$ & 1,00 \\
4. & $"$ & 1,00 \\
5. & $"$ & 2,04
\end{tabular}

In Fig. 6 sind unter Zugrundelegen der Eichkurve Fig. 2 die ge-

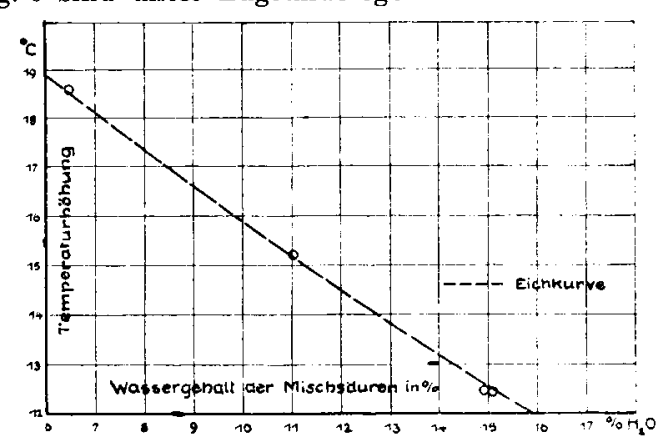

Fig. 6.

fundenen Werte eingetragen. Es geht aus diesen Versuchen hervor, daß ein Gehalt an organischer Substanz die Versuchsergebnisse nicht beeinflußt.

Darmstadt, im September 1921

Chemisch-technisches und elektrochemisches Institut der Technischen Hochschule.

[A. 221.]

\section{Zur Geschichte der Alchemie.}

Von Geh.-Rat Prof. Dr. EilHard Wiedsiaxis in Erlangen.

(Eingeg. 23./9. 1921.)

(Schluß von Seite 523.)

\section{IV. Über chemische Apparate bei den Arabern. .}

In einer früheren Arbeit (Beitr. aus der Geschichte der Chemie von P. Diergart S. 234) habe ich einige Angaben über chemische Apparate veröffentlicht. Das folgende soll diese ergänzen. In der oben erwähnten Gothaer Handschrift (Nr. 1347) [s. S. 522] sind verschiedene Gefäße erwähnt. Zunächst kommt häufig vor ein Topf ('agûz, wörtlich auch altes Weib), in dessen Innerem (baṭ) die Substanzen gemischt werden, dann werden drei- und viereckige und chinesische Gefäße genannt. Eigentümliche Ausdrücke sind Becher (qadah) des Trocknens (tagfiff) und Verwesens (ta'fîn), sowie Flaschen (qarûra) und Brunnen (bi r) des Verwesens. Es bedeutet dies wohl nur, dats in diesen Vorrichtungen die Substanzen trockneten und verwestent. Die letztere Operation spielt ja in der Alchemie eine besondere Rolle.

Ferner werden zahlreiche Öfen (atûn oder tannûr) erwähnt, so ein Ofen mit gemäßigtem Feuer (mu'tadil al nâr); erhitzt wird nicht nur im Ofen, sondern auch auf der Platte (balât). In dem Ofen des Glases oder Glasers (zagâg oder zaggâg) brennt das Feuer der Glaser: es ist wohl das heißeste, das vorkommt: man schmilzt in ihm Mischungen, die vorher im dritten Feuer erhitzt sind und es werden nur noch ein erstes und viertes Feuer genannt. Hierher gehört auch der große Ofen des Email (mînâ). Außerdem gibt es Öfen der Mârijâa, 